\title{
CONSTANT RATIO CURVES IN MINKOWSKI 3-SPACE $\mathbb{E}_{1}^{3}$
}

\author{
GÜNAY ÖZTÜRK, KADRI ARSLAN, AND İLIM KIŞ̧i
}

\begin{abstract}
In the present paper, we consider a curve whose position vector can be written as a linear combination of its Serret-Frenet vectors in Minkowski 3 -space $\mathbb{E}_{1}^{3}$. In particular, we study the non-null curves in $\mathbb{E}_{1}^{3}$ and characterize such curves in terms of their curvature functions. Further, we obtain some results of $T$-constant and $N$-constant type non-null curves in Minkowski 3 -space $\mathbb{E}_{1}^{3}$.
\end{abstract}

\section{INTRODUCTION}

A curve $x: I \subset \mathbb{R} \rightarrow \mathbb{E}^{3}$ in Euclidean 3-space is called a twisted curve if it has nonzero Frenet curvatures $\kappa_{1}(s)$ and $\kappa_{2}(s)$. From the elementary differential geometry, it is well known that a curve $x(s)$ in $\mathbb{E}^{3}$ lies on a sphere if its position vector (denoted also by $x$ ) lies on its normal plane at each point. If the position vector $x$ lies on its rectifying plane, then $x(s)$ is called rectifying curve [4]. Rectifying curves are characterized by the simple equation

$$
x(s)=\lambda(s) T(s)+\mu(s) N_{2}(s),
$$

where $\lambda(s)$ and $\mu(s)$ are smooth functions and $T(s)$ and $N_{2}(s)$ are tangent and binormal vector fields of $x$ respectively [4]. In the same paper, B. Y. Chen gave a simple characterization of rectifying curves. In particular, it is shown in [7] that there exists a simple relation between rectifying curves and centrodes, which play an important role in mechanics kinematics as well as in differential geometry in defining the curves of constant procession. It is also provided that a twisted curve is congruent to a non constant linear function of $s$ [4]. Further, in the Minkowski 3-space $\mathbb{E}_{1}^{3}$, the rectifying curves are investigated in $([9],[15],[17])$. In $[17]$, a characterization of the spacelike, the time-like and the null rectifying curves in the Minkowski 3-space in terms of centrodes is given. For a study on rectifying curves in the dual Lorentzian space $\mathrm{D}_{1}^{3}$ see [20]. For the characterization of rectifying curves

2010 Mathematics Subject Classification. Primary: 53A04, Secondary: 53A05.

Key words and phrases. Position vector, rectifying curve, $W$-curve. 
in three dimensional compact Lee groups or in dual spaces see [21] or [1] respectively.

For any curve $x(s)$ in $\mathbb{E}_{t}^{n}$ with index $t$, the position vector $x$ can be decomposed into its tangential and normal components at each point:

$$
x=x^{T}+x^{N} .
$$

A non-null curve $x(s)$ in $\mathbb{E}_{t}^{n}$ is said to be of constant ratio if the ratio $\left\|x^{T}\right\|:\left\|x^{N}\right\|$ is constant on $x(I)$ where $\left\|x^{T}\right\|$ and $\left\|x^{N}\right\|$ denote the length of $x^{T}$ and $x^{N}$, respectively [2].

Moreover, a curve in $\mathbb{E}_{t}^{n}$ is called $T$-constant (resp. $N$-constant) if the tangential component $x^{T}$ (resp. the normal component $x^{N}$ ) of its position vector $x$ is of constant length [3]. Recently, in [12], the authors give the necessary and sufficient conditions for generic curves in Euclidean 3-space $\mathbb{E}^{3}$ to become $T$-constant and $N$-constant. See also [13] for the results of $T$-constant and $N$-constant curves in Euclidean 4 -space $\mathbb{E}^{4}$.

In the present study, we give a generalization of the rectifying curves in Minkowski 3-space $\mathbb{E}_{1}^{3}$. We consider a non-null curve in $\mathbb{E}_{1}^{3}$ whose position vector satisfies the parametric equation

$$
x(s)=m_{0}(s) T(s)+m_{1}(s) N_{1}(s)+m_{2}(s) N_{2}(s),
$$

for some differentiable functions, $m_{i}(s), 0 \leq i \leq 2$. If $m_{1}(s)=0$ then $x(s)$ becomes a rectifying curve (see, $[15])$. We characterize the twisted curves in terms of their curvature functions $m_{i}(s)$ and give the necessary and sufficient conditions for non-null curves to become $T$-constant and $N$ constant. We give the necessary and sufficient conditions for non-null curves in $\mathbb{E}_{1}^{3}$ to become $W$-curves.

\section{BAsic Notations}

Let $\mathbb{E}_{t}^{n}$ denote the pseudo-Euclidean $n$-space with index $t$. Then the pseudo-Euclidean metric on $\mathbb{E}_{t}^{n}$ is given by

$$
g=-\sum_{i=1}^{t} d x_{i}^{2}+\sum_{j=t+1}^{n} d x_{j}^{2},
$$

where $\left(x_{1}, \ldots, x_{n}\right)$ is a rectangular coordinate system of $\mathbb{E}_{t}^{n}$. In particular $\mathbb{E}_{1}^{n}$ is known as the Lorentzian-Minkowski space-time.

For given positive number $\mathrm{r}$, we put

$$
\mathbb{S}_{t}^{n-1}\left(r^{2}\right)=\left\{x \in \mathbb{E}_{t}^{n}: g(x, x)=r^{2}\right\},
$$

and

$$
\mathbb{H}_{t-1}^{n-1}\left(-r^{2}\right)=\left\{x \in \mathbb{E}_{t}^{n}: g(x, x)=-r^{2}\right\}
$$


It is known that $\mathbb{S}_{t}^{n-1}\left(r^{2}\right)$ and $\mathbb{H}_{t-1}^{n-1}\left(-r^{2}\right)$ are called pseudo-Riemannian and Pseudo-hyperbolic spaces respectively. In particular, $\mathbb{S}_{1}^{n-1}\left(r^{2}\right)$ is called a de Sitter space-time and $\mathbb{H}_{1}^{n-1}\left(-r^{2}\right)$ is an anti-de Sitter space-time [8]. The hyperbolic space $\mathbb{H}^{n-1}\left(-r^{2}\right)$ is defined by

$$
\mathbb{H}^{n-1}\left(-r^{2}\right)=\left\{x \in \mathbb{E}_{t}^{n}: g(x, x)=-r^{2} \text { and } x_{1}>0\right\} .
$$

Recall that an arbitrary vector $v \in \mathbb{E}_{t}^{n}$ is called space-like if $g(v, v)>0$ or $v=0$, time-like if $g(v, v)<0$, and null (light-like) if $g(v, v)=0$ and $v \neq 0$. The norm of a vector $v$ is given by $\|v\|=\sqrt{|g(v, v)|}$, and two vectors $v$ and $w$ are said to be orthonormal, if $g(v, w)=0$. Further, an arbitrary curve $x(s)$ of $\mathbb{E}_{t}^{n}$ is called space-like, time-like or null if its velocity vector $x^{\prime}(s)$ is space-like, time-like or null, respectively [19]. A space-like or time-like curve (i.e., non-null curve) has unit speed, if $g\left(x^{\prime}(s), x^{\prime}(s)\right)= \pm 1$. The light cone $\mathcal{L C}$ of $\mathbb{E}_{t}^{n}$ defined to be

$$
\mathcal{L C}=\left\{x \in \mathbb{E}_{t}^{n}, g(x, x)=0\right\} .
$$

Let $x: I \subset \mathbb{R} \rightarrow \mathbb{E}_{1}^{3}$ be a non-null curve given with arclength function $s$ in Minkowski 3-space $\mathbb{E}_{1}^{3}$. Let us denote $T(s)=x^{\prime}(s)$ and call $T(s)$ as tangent vector of $x$ at $s$. We assume that $T^{\prime}(0)=0$ everywhere. Then we can introduce a unique normal vector field $N_{1}$ and a function $\kappa_{1}$ such that $T^{\prime}(s)=\epsilon_{2} \kappa_{1}(s) N_{1}(s)$, where $\epsilon_{2}=g\left(N_{1}(s), N_{1}(s)\right)$. Since $T(s)$ is a constant length vector field, $N_{1}(s)$ is orthogonal to $T(s)$. The unit binormal vector field of $x$ is defined by $N_{2}(s)=T(s) \times N_{1}(s)$ which is a unique vector field orthogonal to both $T(s)$ and $N_{1}(s)$. One can define a function $\kappa_{2}$ by the equation $N_{2}^{\prime}(s)=-\epsilon_{2} \kappa_{2}(s) N_{1}(s)$. Then the famous Serret-Frenet equations in Minkowski 3-space $\mathbb{E}_{1}^{3}$ are given by

$$
\begin{aligned}
T^{\prime}(s) & =\epsilon_{2} \kappa_{1}(s) N_{1}(s), \\
N_{1}^{\prime}(s) & =-\epsilon_{1} \kappa_{1}(s) T(s)-\epsilon_{1} \epsilon_{2} \kappa_{2}(s) N_{2}(s), \\
N_{2}^{\prime}(s) & =-\epsilon_{2} \kappa_{2}(s) N_{1}(s),
\end{aligned}
$$

where $\kappa_{1}(s)$ and $\kappa_{2}(s)$ are the first and second curvatures of the curve $x$ and

$$
\begin{gathered}
\epsilon_{1}=g(T(s), T(s))= \pm 1, \epsilon_{2}=g\left(N_{1}(s), N_{1}(s)\right)= \pm 1, \\
g\left(N_{2}(s), N_{2}(s)\right)=-\epsilon_{1} \epsilon_{2} .
\end{gathered}
$$

If the Frenet curvature $\kappa_{1}(s)$ and torsion $\kappa_{2}(s)$ of $x$ are constant functions, then $x$ is called a screw line or a helix [10], [11]. Since these curves are the traces of 1-parameter family of the groups of Euclidean transformations, F. Klein and S. Lie called them $W$-curves [18]. 


\section{Characterization of Non-null Curves in $\mathbb{E}_{1}^{3}$}

In the present section, we characterize the non-null curves given with arclength function $s$ in $\mathbb{E}_{1}^{3}$ in terms of their curvatures. Let $x: I \subset \mathbb{R} \rightarrow \mathbb{E}_{1}^{3}$ be a unit speed regular curve with curvatures $\kappa_{1}(s) \neq 0$ and $\kappa_{2}(s)$. By definition, the position vector of the curve (also defined by $x$ ) satisfies the vectorial equation (3) for some differential functions $m_{i}(s), 0 \leq i \leq 2$. Differentiating (3) with respect to arclength parameter $s$ and using the Serret-Frenet equations (4), we obtain

$$
\begin{aligned}
x^{\prime}(s)= & \left(m_{0}^{\prime}(s)-\epsilon_{1} \kappa_{1}(s) m_{1}(s)\right) T(s) \\
& +\left(m_{1}^{\prime}(s)+\epsilon_{2} \kappa_{1}(s) m_{0}(s)-\epsilon_{2} \kappa_{2}(s) m_{2}(s)\right) N_{1}(s) \\
& +\left(m_{2}^{\prime}(s)-\epsilon_{1} \epsilon_{2} \kappa_{2}(s) m_{1}(s)\right) N_{2}(s) .
\end{aligned}
$$

It follows that

$$
\begin{aligned}
m_{0}^{\prime}-\epsilon_{1} \kappa_{1} m_{1} & =1, \\
m_{1}^{\prime}+\epsilon_{2} \kappa_{1} m_{0}-\epsilon_{2} \kappa_{2} m_{2} & =0 \\
m_{2}^{\prime}-\epsilon_{1} \epsilon_{2} \kappa_{2} m_{1} & =0 .
\end{aligned}
$$

Non-null $W$-curves in $\mathbb{E}_{1}^{3}$ were characterized by Ilarslan and Boyacıoğlu in [14]. As a consequence of (6) the following result explicitly determines all the non-null $W$-curves in $\mathbb{E}_{1}^{3}$. Observe that, this result gives a generalization of the results in [14].

Proposition 1. Let $x: I \subset \mathbb{R} \rightarrow \mathbb{E}^{3}$ be a non-null curve given with arclength function $s$. If $x$ is a $W$-curve of $\mathbb{E}_{1}^{3}$, then

$$
\begin{gathered}
m_{0}=\epsilon_{1} \kappa_{1} \int m_{1} d s+(s+c), \\
m_{2}=\epsilon_{1} \epsilon_{2} \kappa_{2} \int m_{1} d s,
\end{gathered}
$$

where $m_{1}$ is the solution of

$$
m_{1}^{\prime \prime}+\epsilon_{1}\left(\epsilon_{2} \kappa_{1}^{2}-\kappa_{2}^{2}\right) m_{1}+\epsilon_{2} \kappa_{1}=0 .
$$

\subsection{Curves of Constant-ratio.}

Definition 1. Let $x: I \subset \mathbb{R} \rightarrow \mathbb{E}_{t}^{n}$ be a non-null unit speed curve in pseudoRiemannian space $\mathbb{E}_{t}^{n}$. Then the position vector $x$ can be decomposed into its tangential and normal components at each point as in (2). If the ratio $\left\|x^{T}\right\|:\left\|x^{N}\right\|$ is constant on $x(I)$, then $x$ is said to be of constant-ratio [2].

For a unit speed space-like curve $x$ in $\mathbb{E}_{t}^{n}$, the gradient of the distance function $\rho=\|x(s)\|$ is given by

$$
\operatorname{grad} \rho=\frac{d \rho}{d s} x^{\prime}(s)=\frac{<x(s), x^{\prime}(s)>}{\|x(s)\|} T(s),
$$


where $T$ is the tangent vector field of $x$.

The following examples are known.

Example 1. For any real numbers $a$ and $c$ with $0<c<1$, the curve

$x(s)=\left(a s, \sqrt{c^{2}+a^{2}} s \sin \left(\frac{\sqrt{1-c^{2}}}{\sqrt{c^{2}+a^{2}}} \ln s\right), \sqrt{c^{2}+a^{2}} s \cos \left(\frac{\sqrt{1-c^{2}}}{\sqrt{c^{2}+a^{2}}} \ln s\right)\right)$

in $\mathbb{E}_{1}^{3}$ is a unit speed space-like curve satisfying $\|\operatorname{grad} \rho\|=c<1$ (see, [6]).

Example 2. For any real numbers $a$ and $c$ satisfying $c>1$ and $c>a \leq 0$.

Then the curve

$$
x(s)=\left(\frac{1}{2} s^{1-\lambda}\left(s^{2 \lambda}-b^{2}\right), \frac{1}{2} s^{1-\lambda}\left(s^{2 \lambda}+b^{2}\right), a s\right)
$$

in $\mathbb{E}_{1}^{3}$ is a unit speed space-like curve satisfying $\|\operatorname{grad} \rho\|=c>1$, where $b=\sqrt{c^{2}+a^{2}}$ and $\lambda=\frac{\sqrt{c^{2}-1}}{b}$ (see, [6]).

Lemma 1. [6] Let $x: I \subset \mathbb{R} \rightarrow \mathbb{E}_{t}^{n}$ be a unit speed non-null curve in $\mathbb{E}_{t}^{n}$ with index $t$. Then $\|\operatorname{grad} \rho\|=c$ holds for a constant $c$ if and only if, up to translation of the arclength function $s$, we have $\|x(s)\|=c s$.

Theorem 1. [5] Let $x: I \subset \mathbb{R} \rightarrow \mathbb{E}_{t}^{n}$ be a unit speed space-like curve in $\mathbb{E}_{t}^{n}$ with index $t$. Then $\|\operatorname{grad} \rho\|=c$ holds for a constant $c$ if and only if one of the following eight cases occurs:

i) $x$ lies in the light-like cone $\mathcal{L C}$.

ii) $x$ lies in a pseudo-Riemannian sphere $\mathbb{S}_{t}^{n-1}\left(r^{2}\right)$.

iii) $x$ lies in a pseudo-hyperbolic space $\mathbb{H}_{t-1}^{n-1}\left(-r^{2}\right)$.

iv) $x$ lies on open portion of a space-like line through the origin.

$v)$ There exist a real number $b>1$ and time-like unit speed curve $y=y(u)$ which lies in the unit pseudo-Riemannian sphere $\mathbb{S}_{t}^{n-1}(1)$ such that $x$ is given by $x(s)=b s y\left(\frac{\sqrt{b^{2}-1}}{b} \ln s\right)$.

vi) There exist a real number $b \in(0,1)$ and space-like unit speed curve $y=y(u)$ which lies in the unit pseudo-Riemannian sphere $\mathbb{S}_{t}^{n-1}(1)$ such that $x$ is given by $x(s)=b s y\left(\frac{\sqrt{1-b^{2}}}{b} \ln s\right)$.

vii) There exist a null curve $y=y(s)$ lying in the unit pseudo-Riemannian $\mathbb{S}_{t}^{n-1}(1)$ such that $x$ is given by $x(s)=b s y(s)$.

viii) There exist a real number $b>0$ and space-like unit speed curve $y=w(u)$ which lies in the unit pseudo-hyperbolic space $\mathbb{H}_{t-1}^{n-1}(1)$ such that $x$ lies given by $x(s)=b s w\left(\frac{\sqrt{1-b^{2}}}{b} \ln s\right)$.

The following results characterize constant-ratio curves in $\mathbb{E}_{1}^{3}$. 
Proposition 2. Let $x: I \subset \mathbb{R} \rightarrow \mathbb{E}_{1}^{3}$ be a unit speed non-null curve in $\mathbb{E}_{1}^{3}$. If $x$ is of constant-ratio then the position vector of the curve has the parametrization of the form

$$
x(s)=\frac{c^{2} s}{\epsilon_{1}} T(s)+\left(\frac{c^{2}-\epsilon_{1}}{\kappa_{1}}\right) N_{1}(s)+\left(\frac{\kappa_{1} c^{2} s}{\epsilon_{1} \kappa_{2}}+\frac{\left(\epsilon_{1}-c^{2}\right) \kappa_{1}^{\prime}}{\epsilon_{2} \kappa_{2} \kappa_{1}^{2}}\right) N_{2}(s),
$$

where $c$ is a real constant.

Proof. Let $x$ be a non-null curve of constant-ratio given with arclength function $s$. Then, from the previous result, the distance function $\rho$ of $x$ satisfies the equality $\rho=\|x(s)\|=c s$ for some real constant $c$. Further, using (7), we get

$$
\|\operatorname{grad} \rho\|=\frac{<x(s), x^{\prime}(s)>}{\|x(s)\|}=c .
$$

Since $x$ is a non-null curve of $\mathbb{E}_{1}^{3}$, then it satisfies the equality (3). Thus, by the use of (8) and (6) with the previous lemma, we get

$$
\begin{aligned}
& m_{0}(s)=\frac{c^{2} s}{\epsilon_{1}} \\
& m_{1}(s)=\frac{c^{2}-\epsilon_{1}}{\kappa_{1}}, \\
& m_{2}(s)=\frac{\kappa_{1} c^{2} s}{\epsilon_{1} \kappa_{2}}+\frac{\left(\epsilon_{1}-c^{2}\right) \kappa_{1}^{\prime}}{\epsilon_{2} \kappa_{2} \kappa_{1}^{2}} .
\end{aligned}
$$

Substituting these values into (3), we obtain the desired result.

As a consequence of (9) with the third equation of (6), we get the following result.

Corollary 1. Let $x: I \subset \mathbb{R} \rightarrow \mathbb{E}_{1}^{3}$ be a unit speed non-null curve in $\mathbb{E}_{1}^{3}$. Then $x$ is of constant-ratio if and only if

$$
\left(\frac{\kappa_{1} c^{2} s}{\epsilon_{2} \kappa_{2}}+\frac{\left(\epsilon_{1}-c^{2}\right) \kappa_{1}^{\prime}}{\epsilon_{1} \kappa_{2} \kappa_{1}^{2}}\right)^{\prime}+\frac{\kappa_{2}}{\kappa_{1}}\left(\epsilon_{1}-c^{2}\right)=0 .
$$

\subsection{T-constant Curves in $\mathbb{E}_{1}^{3}$.}

Definition 2. Let $x: I \subset \mathbb{R} \rightarrow \mathbb{E}_{t}^{n}$ be a unit speed non-null curve in $\mathbb{E}_{t}^{n}$. If $\left\|x^{T}\right\|$ is constant, then $x$ is called a T-constant curve [6]. Further, a $T$-constant curve $x$ is called first kind if $\left\|x^{T}\right\|=0$, otherwise second kind.

As a consequence of (3) with (6) we get the following result.

Lemma 2. Let $x: I \subset \mathbb{R} \rightarrow \mathbb{E}_{1}^{3}$ be a unit speed non-null curve in $\mathbb{E}_{1}^{3}$. Then $x$ is a T-constant curve if and only if

$$
\begin{aligned}
0 & =1+\epsilon_{1} \kappa_{1} m_{1}, \\
m_{1}^{\prime} & =\epsilon_{2} \kappa_{2} m_{2}-\epsilon_{2} \kappa_{1} m_{0},
\end{aligned}
$$




$$
m_{2}^{\prime}=\epsilon_{1} \epsilon_{2} \kappa_{2} m_{1}
$$

hold, where $m_{0} \in \mathbb{R}, m_{1}(s)$ and $m_{2}(s)$ are differentiable functions.

Thus, from this definition, it is easy to see that the non-null $T$-constant curves of first kind can be characterized by the following equations.

As a consequence of (10), we get the following result.

Theorem 2. Let $x: I \subset \mathbb{R} \rightarrow \mathbb{E}_{1}^{3}$ be a unit speed non-null curve in $\mathbb{E}_{1}^{3}$ with the curvatures $\kappa_{1}>0$ and $\kappa_{2} \neq 0$. Then $x$ is a T-constant curve of first kind if and only if

$$
\frac{\varepsilon_{1} \kappa_{2}}{\kappa_{1}}+\left(\frac{\kappa_{1}^{\prime}}{\kappa_{1}^{2} \kappa_{2}}\right)^{\prime}=0
$$

Proof. Let $x$ be a $T$-constant curve of first kind. Then, from the second and third equalities in (10), we get $m_{2}=\frac{m_{1}^{\prime}}{\varepsilon_{2} \kappa_{2}}$ and $m_{2}^{\prime}-\varepsilon_{1} \varepsilon_{2} m_{1} \kappa_{2}=0$. Further, substituting the differentiation of the first equation and $m_{1}=-\frac{1}{\varepsilon_{1} \kappa_{1}}$ into the third equation, we get the result.

Remark 1. Every T-constant curve of the first kind is normal curve in $\mathbb{E}_{1}^{3}$. Observe that, the time-like and null normal curves in Minkowski space $\mathbb{E}_{1}^{3}$ are classified by Ilarslan and Nesovic in [16].

As a consequence of Theorem 1, we get the following result.

Corollary 2. Let $x: I \subset \mathbb{R} \rightarrow \mathbb{E}_{1}^{3}$ be a unit speed non-null curve in $\mathbb{E}_{1}^{3}$ with the curvatures $\kappa_{1}>0$ and $\kappa_{2} \neq 0$. Then $x$ is a T-constant curve of first kind if and only if either $x$ lies on a pseudo-sphere $S^{2}(r)$ of $\mathbb{E}_{1}^{3}$ (see, [16]) or on a pseudo-hyperbolic space $H^{2}(r)$ of $\mathbb{E}_{1}^{3}$.

By the use of (10) with (11), we can construct the following example.

Example 3. The unit speed non-null curve given with the parametrization

$$
x(s)=\cosh \left(\int \varepsilon_{2} \kappa_{2} d s\right) N_{1}(s)+\varepsilon_{1} \sinh \left(\int \varepsilon_{2} \kappa_{2} d s\right) N_{2}(s)
$$

is a $T$-constant curve of first kind in $\mathbb{E}_{1}^{3}$.

As a consequence of (10), we get the following result.

Theorem 3. Let $x: I \subset \mathbb{R} \rightarrow \mathbb{E}_{1}^{3}$ be a non-null unit speed curve in $\mathbb{E}_{1}^{3}$. Then $x$ is a T-constant curve of second kind if and only if

$$
\left(\frac{\kappa_{1}^{\prime}+\varepsilon_{1} \varepsilon_{2} m_{0} \kappa_{1}^{3}}{\kappa_{1}^{2} \kappa_{2}}\right)^{\prime}+\frac{\varepsilon_{1} \kappa_{2}}{\kappa_{1}}=0
$$

holds for some constant function $m_{0}$. 
Proof. Suppose that $x$ is a $T$-constant curve of second kind. Then, by the use of $(10)$, we get

$$
0=m_{2}^{\prime}-\varepsilon_{1} \varepsilon_{2} m_{1} \kappa_{2}, m_{2}=\frac{m_{1}^{\prime}+\varepsilon_{2} \kappa_{1} m_{0}}{\varepsilon_{2} \kappa_{2}} .
$$

Further, substituting the differentiation of second equation and using $m_{1}=$ $-\frac{1}{\varepsilon_{1} \kappa_{1}}$ with first equation, we get the result.

Corollary 3. Let $x \in \mathbb{E}_{1}^{3}$ be a unit speed non-null curve in $\mathbb{E}_{1}^{3}$. If $x$ is a T-constant curve of second kind with non-zero constant first curvature $\kappa_{1}$, then

$$
\kappa_{2}(s)=\mp \frac{\sqrt{a}}{\sqrt{c_{1} a-2 \varepsilon_{2} s}}
$$

holds for some constant functions $c_{1}$ and $a=\kappa_{1}^{2} m_{0}$.

Proof. Suppose, first curvature $\kappa_{1}$ is a constant function. Then by the use of (13), we get

$$
m_{0} \kappa_{1}\left(\frac{1}{\kappa_{2}}\right)^{\prime}+\frac{\varepsilon_{2} \kappa_{2}}{\kappa_{1}}=0
$$

which has a non-trivial solution (15).

For $T$-constant curves of second kind, we give the following results.

Proposition 3. Let $x \in \mathbb{E}_{1}^{3}$ be a unit speed non-null T-constant curve of second kind. Then the distance function $\rho=\|x\|$ satisfies

$$
\rho= \pm \sqrt{c_{1} s+c_{2}}
$$

for some constants $c_{1}=2 \varepsilon_{1} m_{0}$ and $c_{2}$.

Proof. Let $x \in \mathbb{E}_{1}^{3}$ be a $T$-constant tight curve of second kind. Then, by definition, the curvature function $m_{0}(s)$ of $x$ is constant. Hence, differentiating the squared distance function $\rho^{2}=\langle x(s), x(s)\rangle$ and using (7), we get $\rho \rho^{\prime}=\varepsilon_{1} m_{0}$. It is an easy calculation to show that, this differential equation has a nontrivial solution (16).

\subsection{N-constant Curves in $\mathbb{E}_{1}^{3}$.}

Definition 3. Let $x: I \subset \mathbb{R} \rightarrow \mathbb{E}_{t}^{n}$ be a unit speed non-null curve in $\mathbb{E}_{t}^{n}$. If $\left\|x^{N}\right\|$ is constant, then $x$ is called an $N$-constant curve. For an $N$-constant curve $x$, either $\left\|x^{N}\right\|=0$ or $\left\|x^{N}\right\|=\mu$ for some non-zero smooth function $\mu[6]$. Further, an $N$-constant curve $x$ is called first kind if $\left\|x^{N}\right\|=0$, otherwise second kind. 
Note that, for an $N$-constant curve $x$ in $\mathbb{E}_{1}^{3}$

$$
\left\|x^{N}(s)\right\|^{2}=\varepsilon_{2} m_{1}^{2}(s)-\varepsilon_{1} \varepsilon_{2} m_{2}^{2}(s),
$$

becomes a constant function.

For the $N$-constant curves of first kind, we give the following result.

Theorem 4. Let $x: I \subset \mathbb{R} \rightarrow \mathbb{E}_{1}^{3}$ be a unit speed non-null curve in $\mathbb{E}_{1}^{3}$. Then $x$ is an $N$-constant curve of first kind if and only if either $x$ is congruent to a pseudo-line or a T-constant curve of first kind with $x(s)=\frac{-1}{\kappa_{1}(s)}\left(N_{1}+N_{2}\right)$.

Proof. Suppose that $x$ is an $N$-constant curve of first kind in $\mathbb{E}_{1}^{3}$, then $m_{1}^{2}-\varepsilon_{1} m_{2}^{2}=0$. Further, if $x$ is a time-like curve, then $m_{1}=m_{2}=0$ and $m_{0}=s+b$, which implies that $\kappa_{1}=\kappa_{2}=0$. Consequently, $x$ is a pseudo-line in $\mathbb{E}_{1}^{3}$. Moreover, if $x$ is a space-like curve, then $m_{1}= \pm m_{2}$. In particular, if $m_{1}=m_{2}$, then from (6) $m_{1}=m_{2}=\frac{-1}{\kappa_{1}}$. This means that $x$ is a $T$-constant curve of first kind which has the parametrization of the form $x(s)=\frac{-1}{\kappa_{1}(s)}\left(N_{1}+N_{2}\right)$. Similarly, for $m_{1}=-m_{2}$, we obtain the same results. The proof of the converse statement is trivial.

Definition 4. A space curve $x: I \subset \mathbb{R} \rightarrow \mathbb{E}_{1}^{3}$ whose position vector always lies in its rectifying plane is called a rectifying curve. Thus, for a rectifying curve $x: I \subset \mathbb{R} \rightarrow \mathbb{E}_{1}^{3}$, the position vector $x(s)$ satisfies the simple equation

$$
x(s)=m_{0}(s) T(s)+m_{2}(s) N_{2}(s)
$$

for some differentiable functions $m_{0}(s)$ and $m_{2}(s)$ [4]. For more details for rectifying curves in $\mathbb{E}_{1}^{3}$ see also [15] and [9].

The following results provides some simple characterizations of non-null rectifying curves in $\mathbb{E}_{1}^{3}$.

Theorem 5. [15] Let $x: I \subset \mathbb{R} \rightarrow \mathbb{E}_{1}^{3}$ be a non-null rectifying curve with $\kappa_{1} \neq 0$, and let $s$ be its arclength parameter. Then

i) The tangential component of the position vector of the curve $x(s)$ is given by $\langle x(s), T\rangle=\varepsilon_{1}(s+b)$, for some real constant $b$.

ii) The distance function $\rho=\|x(s)\|$ satisfies $\rho^{2}=(s+b) \varepsilon_{1}-a \varepsilon_{1} \varepsilon_{2}$ for some real constants $a$ and $b$.

iii) The normal component of the position vector of the curve has constant length and the distance function $\rho$ is non constant.

$i v)$ The torsion $\kappa_{2}(s) \neq 0$ and the binormal component of the position vector of the curve is constant, i.e. $\left\langle x, N_{2}\right\rangle$ is constant.

Conversely, if $x(s)$ is a unit speed non-null curve in $\mathbb{E}_{1}^{3}$, with space-like or time-like rectifying plane, the curvature $\left.\kappa_{1}(s)\right\rangle 0,\langle T, T\rangle=\varepsilon_{1}= \pm 1$ 
and one of the statements $(i),(i i),(i i i)$ and $(i v)$ holds, then $x$ is a rectifying curve.

Theorem 6. [15] $x: I \subset \mathbb{R} \rightarrow \mathbb{E}_{1}^{3}$ be a non-null curve in $\mathbb{E}_{1}^{3}$ with $\kappa_{1} \neq 0$. Then $x$ is congruent to a rectifying curve if and only if the ratio of torsion and curvature of the curve is a linear function of arclength function s, i.e. $\frac{\kappa_{2}}{\kappa_{1}}(s)=c_{1} s+c_{2}$ for some constants $c_{1}$ and $c_{2}$.

We obtain the following result.

Theorem 7. Let $x(s)$ be a non-null curve in $\mathbb{E}_{1}^{3}$ and $s$ be its arclength function. Then $x$ is an $N$-constant curve of second kind if and only if either $x$ is congruent to a rectifying curve

$$
x(s)=(s+b) T(s)+a N_{2}(s), \quad a, b \in \mathbb{R},
$$

with

$$
\frac{\kappa_{2}}{\kappa_{1}}(s)=\frac{s+b}{a},
$$

or, congruent to a T-constant curve of first kind given with the parametrization

$$
x(s)=\frac{-1}{\varepsilon_{1} \kappa_{1}(s)} N_{1}(s)+\frac{\kappa_{1}^{\prime}(s)}{\varepsilon_{1} \varepsilon_{2} \kappa_{1}^{2}(s) \kappa_{2}(s)} N_{2}(s), \quad a, b \in \mathbb{R},
$$

such that the equality (11) holds.

Proof. Let $x$ be an $N$-constant curve of second kind, then the equation $m_{1} m_{1}^{\prime}-\varepsilon_{1} m_{2} m_{2}^{\prime}=0$ holds. Hence, by the use of the equations in (6), we get

$$
m_{1}\left(m_{1}^{\prime}-\varepsilon_{2} \kappa_{2} m_{2}\right)=0 .
$$

So, there are two possible cases: $m_{1}=0$ or $m_{1}^{\prime}-\varepsilon_{2} \kappa_{2} m_{2}=0$. Moreover, if $m_{1}=0$, then $m_{0}=s+b, m_{2}=a$ for some constant functions $a$ and $b$. Hence, $x$ becomes a rectifying curve with the parametrization of the form (18). Substituting these values into second equation of (6), we get (19). Furthermore, if $m_{1}^{\prime}-\varepsilon_{2} \kappa_{2} m_{2}=0$ holds, then using the second equality in (6), we get $\kappa_{1}=0$, or $m_{0}=0$. Note that, if $\kappa_{1}=0$ then $x$ is congruent to a pseudo-line which is an $N$-constant curve of first kind. So this case does not occur. For the case $m_{0}=0$, we get $m_{1}=\frac{-1}{\varepsilon_{1} \kappa_{1}(s)}, m_{2}=\frac{\kappa_{1}^{\prime}(s)}{\varepsilon_{1} \varepsilon_{2} \kappa_{1}^{2}(s) \kappa_{2}(s)}$. Hence, $x$ becomes a T-constant curve of first kind with the parametrization of the form (20). The proof of the converse statement is trivial. 


\section{REFERENCES}

[1] Z. Bozkurt, I. Gök, O. Z. Okuyucu and F. N. Ekmekci, characterization of rectifying, normal and osculating curves in three dimensional compact Lie groups, Life Sci., 10(2013),819-823.

[2] B. Y. Chen, Constant ratio Hypersurfaces, Soochow J. Math., 28(2001), 353-362.

[3] B. Y. Chen, Geometry of Position Functions of Riemannian Submanifolds in PseudoEuclidean Space, J. Geom., 74(2002), 61-77.

[4] B.Y. Chen, When does the position vector of a space curve always lies in its rectifying plane?, Amer. Math. Monthly, 110(2003), 147-152.

[5] B.Y. Chen, Constant-ratio spacelike submanifolds in pseudo-Euclidean space, Houston J. Math. 29(2003), 281-294.

[6] B. Y. Chen, More on convolution of Riemannian manifolds, Beitrage Algebra Geom., 44(2003), 9-24.

[7] B. Y. Chen and F. Dillen, Rectifying curves as centrodes and extremal curves, Bull. Inst. Math. Acedemia Sinica, 33(2005), 77-90.

[8] K.L. Dugal and A. Bejancu, Lightlike submanifolds of semi-Riemannian manifolds and aplications. Kluwer Academic, Dordrecht, 1996.

[9] R. Ezentaş and S. Türkay, Helical versus of rectifying curves in Lorentzian spaces, Dumlıpınar Univ. Fen Bilim. Esti Dergisi 6(2004), 239-244.

[10] A. Gray, Modern differential geometry of curves and surface, CRS Press, Inc., 1993.

[11] H. Gluck, Higher curvatures of curves in Euclidean space, Amer. Math. Monthly, 73(1966), 699-704.

[12] S. Gürpınar, K. Arslan, and G. Öztürk, A Characterization of Constant-ratio Curves in Euclidean 3-space $\mathbb{E}^{3}$, Acta Universitatis Apulensis, 44(2015), 39-51.

[13] S. Gürpınar, K. Arslan, and G. Öztürk, A New Characterization of Curves in Euclidean 4-Space $\mathbb{E}^{4}$, Buletinul Academiei de Stiiente a Republicii Moldova. Matematica, 83(2017), 39-50.

[14] K. Ilarslan and Ö. Boyacıŏlu, Position vectors of a spacelike W-curve in Minkowski space $E_{1}^{3}$, Bull. Korean Math. Soc., 46(2009), 967-978.

[15] K. Ilarslan, E. Nesovic and T. M. Petrovic, Some characterization of rectifying curves in the Minkowski 3-space, Novi Sad J. Math., 32(2003), 23-32.

[16] K. Ilarslan and E. Nesovic, Timelike and null normal curves in Minkowski space $E_{1}^{3}$, Indian J. Pure Appl. Math. 35(2004), 881-888.

[17] K. Ilarslan and E. Nesovic, On rectifying curves as centrodes and extremal curves in the Minkowski 3-space $\mathbb{E}_{1}^{3}$, Novi. Sad. J. Math. 37(2007), 53-64.

[18] F. Klein and S. Lie, Uber diejenigen ebenenen kurven welche durch ein geschlossenes system von einfach unendlich vielen vartauschbaren linearen Transformationen in sich übergehen,Math. Ann. 4(1871), 50-84.

[19] B. O'Neill, Semi-Riemannian Geometry with Application to Relativity, Academic Press, 1983.

[20] E. Özbey and M. Oral, A study on rectifying curves in the dual Lorentzian space $D_{1}^{3}$, Bull. Korean Math. Soc. 46(2009), 429-438.

[21] A. Yücesan, N. Ayyıldız and A.C. Çöken, On rectifying dual space curves, Rev. Math. Comp.,20(2007), 497-506. 
İzmir Democracy University, Arts and Science Faculty,

Department of Mathematics,

TURKEY

E-mail address: gunay.ozturk@idu.edu.tr

ULUDAG UNIVERSITY,

Arts and Science Faculty,

Department of Mathematics,

TURKEY

E-mail address: arslan@uludag.edu.tr

KOCAELI UNIVERSITY,

Arts and Science Faculty,

Department of Mathematics,

TURKEY

E-mail address: ilim.ayvaz@kocaeli.edu.tr 